Notfall Rettungsmed 2009 · 12:225-225

DOI 10.1007/s10049-008-1139-0

Online publiziert: 6. März 2009

(c) Springer Medizin Verlag 2009

\author{
S. Dreesen ${ }^{1} \cdot$ J. Arrich ${ }^{2}$ \\ ${ }^{1}$ Notfallpädagogisches Institut (NPI), Staatl. anerkannte \\ Rettungsassistentenschule, Essen \\ ${ }^{2}$ Universitätsklinik für Notfallmedizin, Allgemeines Krankenhaus, Wien
}

\title{
Therapeutische Hypothermie bei Asystolie
}

\section{Signifikant schlechteres Outcome?}

\section{Meinungsaustausch zum Beitrag}

Arrich J, Behringer W, Holzer M et al (2008)

Therapeutische Hypothermie. Wann, wie schnell, wie lange, welche Methode? Notfall

Rettungsmed 11:453-458

\section{Leserbrief}

\section{S. Dreesen}

Notfallpädagogisches Institut (NPI),

Staatl. anerkannnte Rettungsassistentenschule Essen

Die Autoren des Beitrags „Therapeutische Hypothermie. Wann, wie schnell, wie lange, welche Methode?" in Ausgabe 7/2008 von „Notfall und Rettungsmedizin" schreiben, es gebe derzeit keine Hinweise darauf, dass eine Hypothermie bei Asystolie nicht wirksam sei. Ich möchte hier auf den Beitrag von C. Storm [1] in „Der Kardiologe“ hinweisen, in welchem ein nichtsignifikanter Trend zum schlechteren Outcome in der Hypothermiegruppe bezogen auf das Erwachen nach dem Koma beschrieben wird.

Der Beitrag ist die Zusammenfassung einer Studie von Kim et al. [2] in der Zeitschrift „Circulation“.

\section{Korrespondenzadresse}

\section{S. Dreesen}

Notfallpädagogisches Institut (NPI),

Staatl. annerkannte Rettungsassistentenschule Müller-Breslau-Str. 30a, 45130 Essen

s.dreesen@web.de

\section{Erwiderung}

\section{J. Arrich \\ Universitätsklinik für Notfallmedizin, Allgemeines Krankenhaus, Wien}

Wir danken Herrn Dr. Dreesen für die Diskussionsanregung zum Effekt von Hypothermie bei Asystolie, welches ein durchaus kontroverses Thema ist. Die Studie von Kim et al. [2] ist in diesem Zusammenhang allerdings aus mehreren Gründen kritisch zu sehen:

1. Bereits im Titel wird deutlich, dass es sich um eine Pilotstudie handelt, das primäre Ziel war also keinesfalls die Untersuchung des Outcome, sondern die Überprüfung der Machbarkeit prähospitaler Kühlung. Die Aussagekraft der Studie reicht bei weitem nicht aus, um eine Aussage über das Outcome zu treffen.

2. Wenn man weiter liest, wurden 60 der 97 aufgenommen Patienten unabhängig von der prähospitalen Kühlung auch im Krankenhaus weitergekühlt. Man kann daher eigentlich nicht sagen, ob der (ohnehin nicht signifikante) Effekt der prä- oder der innerhospitalen Kühlung zuzuschreiben ist.

3. Von den 63 Patienten, die zu therapeutischer Hypothermie randomisiert wurden, erhielten nur 12 (!) Patienten die adäquate Menge von 21 kalter Flüssigkeit. Ich frage mich, welchen Effekt man überhaupt messen wollte.
Insgesamt würde ich davon abraten, die Studie von Kim et al. [2] als OutcomeStudie zu zitieren.

\section{Korrespondenzadresse \\ Dr. J. Arrich \\ Universitätsklinik für Notfallmedizin, Allgemeines Krankenhaus Währinger Gürtel 18-20/6D, A-1090 Wien jasmin.arrich@meduniwien.ac.at}

Interessenkonflikt. Die korrespondierenden Autoren geben an, dass kein Interessenkonflikt besteht.

\section{Literatur}

1. Storm C (2008) Einleitung milder therapeutischer Hypothermie nach Reanimation bereits durch den Notarzt? Kardiologe 2:14-16

2. Kim F, Olsufka M, Longstreth WT et al (2007) Pilot randomized clinical trial of prehospital induction of mild hypothermia in out-of-hospital cardiac arrest patients with a rapid infusion of $4^{\circ} \mathrm{C}$ normal saline. Circulation 115:3064-3070 